\title{
Clinical and Microbiological Evaluation of Biofilm-Gingival Interface Classification in Patients With Generalized Forms of Periodontitis
}

\author{
JAN KOWALSKI and RENATA GÓRSKA \\ Medical University of Warsaw, Department of Periodontology and Oral Diseases, Warsaw, Poland
}

Submitted 1 August 2013, revised 23 December 2013, accepted 25 April 2014

Abstract

\begin{abstract}
Study objective: In 2008 a new division of periodontal diseases based on the concept of biofilm-gingival interface (BGI), was presented. The aim of this study was to assess whether the proposed BGI categories could be useful in diagnosing patients with generalized forms of both aggressive (gAP) and chronic (gCP) periodontitis. Design and key methods: The study group consisted of 40 individuals (17 with gAP and 23 with gCP). All patients were divided into three groups according to the BGI scale: 4 patients with P1 (pockets deeper than 4 mms with bleeding index below 10\%), 20 individuals with P2 (pockets deeper than $4 \mathrm{mms}$ with bleeding index 10-50\%) and 16 individuals with P3 (pockets deeper than $4 \mathrm{mms}$ with bleeding index above 50\%). The presence of bacteria in subgingival plaque was analyzed using the polymerase chain reaction method. Results: Statistically significant differences with respect to all parameters measured were found when patients were grouped according to the BGI scale - they were higher in P3 group. In gAP group A. actinomycetemcomitans and C. rectus were found in a higher numbers. No such differences were observed between P2 and P3 groups. Conclusions: The BGI scale seems to be helpful in effective and quick classifying of patients into adequate clinical subgroups.
\end{abstract}

Key words: Periodontal diseases, periodontal pathogens, biofilm, polymerase chain reaction

\section{Introduction}

The current classification of periodontal diseases and conditions has been used since 1999 (Armitage, 1999). The division of periodontal inflammations is based on several clinical criteria. Aggressive periodontitis has been diagnosed in generally healthy patients, with a family history of the disease, in whom the clinical attachment level (CAL) decreases rapidly, and who have relatively small dental biofilm deposits in comparison to the inflammatory reaction. This disease can be also characterized by specific microflora (elevated proportions of Aggregatibacter actinomycetemcomitans and - sometimes - Porphyromonas gingivalis), hyperresponsive phenotype of macrophages (elevated levels of interleukin-1 beta and prostaglandin E2) and tendency to self limiting (Armitage, 1999). Localized and generalized aggressive periodontitis (lAP and gAP, respectively) differ in the number of teeth involved in the destructive periodontal process. The most common periodontal inflammation, chronic periodontitis, can be divided according to its extent and severity. The threshold between localized and generalized chronic periodontitis (ICP and gCP, respectively) is $30 \%$ of sites affected with a loss of clinical attachment. Mild, moder- ate or severe $\mathrm{CP}$ is diagnosed depending on the value of the maximal CAL (Armitage, 1999).

Offenbacher et al. (2007) studied over 6700 individuals with various forms of periodontal disease. Researchers aimed to establish a link between the bacteria constituting dental biofilm and inflammatory process, which occurs in periodontal tissues. After clinical evaluation, titers of specific IgG antibodies in peripheral blood and in gingival crevicular fluid were determined. In conjunction with the results of both the clinical and immunological examination, a retrospective analysis was made, which led to the diversification of patients into 5 groups. Taken under consideration the clinical parameters such as pocket depth (PD) and bleeding on probing (BOP), the differences between groups were further based on the levels of antibodies against periodontal pathogens: BGI-H, individuals with healthy periodontium, in whom the PD did not exceed $4 \mathrm{~mm}$ and the BOP was below $10 \%$; BGI-G, patients suffering from gingivitis, in whom the PD did not exceed $4 \mathrm{~mm}$ and the BOP was over $10 \%$; and 3 groups of patients suffering from deep lesions (DL), signified that at least one site had a PD over $4 \mathrm{~mm}$ : BGI-DL/LB - (marked also as P1), patients with low bleeding (LB), who had BOP of below 10\%;

* Corresponding author: J. Kowalski, Dept of Periodontology and Oral Diseases, ul. Miodowa 18, 00-246 Warsaw, Poland; phone/ fax: (+4822)5022036; e-mail: jkowalski@wum.edu.pl 
BGI-DL/MB - (marked also as P2), patients with moderate bleeding $(\mathrm{MB})$, who had $\mathrm{BOP}$ values ranging from $10 \%$ to $50 \%$; and BGI-DL/SB - (marked also as P3), patients with severe bleeding (SB), who had BOP over $50 \%$ (Offenbacher et al., 2008). Apart from the relevance between the clinical parameters and specific IgG levels, significant changes with respect to the microbial profile were found between those 5 groups. The BGI-G group had significantly more bacteria belonging to the orange complex (according to Socransky et al. (1998)), particularly the Campylobacter rectus species. The P1 group had less bacteria than the BGI-H and BGI-G groups, $\mathrm{P} 2$ had more bacteria from the red complex, and in $\mathrm{P} 3$ group the amount of all bacteria was increased (Offenbacher et al., 2007).

The new approach, matching the effect with the cause, resulted in an apparently simple classification (further called the BGI scale). In this proposition only two periodontal parameters were used- PD and BOP. Both are easy to use and leave little scope or field for mistake or misinterpretation, while the parameter crucial for the currently used classification, i.e. CAL, requires at least some experience with the positioning of the cementum-enamel junction level. Also, in the light of current discussions on the subject of diminishing differences in occurrence of bacteria characteristic for CP (red complex) and AP (A. actinomycetemcomitans and P. gingivalis) (Ximenez-Fyvie et al., 2006), the BGI scale seems to be helpful in describing periodontal status in patients with generalized form of inflammation. Recently, Heller and coworkers (2012) published results of the research conducted on 260 Brazilians suffering from gAP (75 individuals) and CP (185 individuals). In that group, by the means of checkerboard DNA-DNA hybridization technique, counts of over 40 bacterial species (46 strains) were evaluated. Only few species differed between gAP and gCP; Eubacterium nodatum was strongly associated with $\mathrm{gAP}$, while Porphyromonas gingivalis and Treponema denticola were closely related to CP. Those differences may be related to such factors as: race, country of residence, age (Sanz et al., 2000; Faveri et al., 2008). According to the announcement of $6^{\text {th }}$ European Workshop of Periodontology, individual bacteria of the given species may vary significantly in their pathogenity, as only approximately $2 / 3$ of the genes are shared within the species. Moreover, differences were found in the occurrence of the principal periodontal pathogens in the various world regions (Rylev and Kilian, 2008).

The relative simplicity of the BGI scale may be the reason for its weakness, potentially leading to misdiagnosis. Grouping all gingival inflammations into one group - BGI-G, seems to be a daring idea. There is also no trace of systemic diseases being taken into account. But even if the BGI scale is limited to generally healthy subjects, doubts still persist. There is no localized or generalized form of inflammation even though they are differentiated in the currently used classification. In our opinion, the potential weakness is that on this scale only one actual parameter (BOP) describes the extent of the inflammation, while the second one in all three stages of periodontitis $(\mathrm{P} 1, \mathrm{P} 2, \mathrm{P} 3)$ retains the same criteria of classification, i.e. $\mathrm{PD}>4 \mathrm{mms}$. Thus, there is the question whether the BGI scale would be effective in grouping patients with periodontal inflammation. The aim of this study was whether the proposed BGI scale could be a useful clinical tool to diagnose patients with generalized aggressive periodontitis and generalized severe chronic periodontitis on the basis of clinical evaluation and microbiological tests.

\section{Experimental}

\section{Material and Methods}

The study group consisted of 40 patients with a mean age of 38.6 years (range, 23-60 yrs) attending to the Department of Periodontology and Oral Diseases of the Medical University of Warsaw between 2008 and 2009. All patients were enrolled in the study on the basis of the following general inclusion criteria: at least 4 teeth (each in a different dentition quadrant) with a pocket depth in at least one site exceeding 5 millimetres.

Inclusion criteria for the gCP group: extensive deposits of plaque/calculus, over $30 \%$ of all sites affected with CA loss, and with at least one site of CA loss being more than $4 \mathrm{~mm}$. Twenty three patients were enrolled in this group (mean age, $44.7 \mathrm{yrs}$; range 37-60 yrs).

Inclusion criteria for the gAP group: positive family history of periodontal disease (based on the anamnesis), general health, an inflammatory state inadequate to the amount of dental deposits present on the teeth, disease progression intensified in the areas of the central incisors and/or first molars. At least three other teeth affected by periodontal inflammation. In this group 17 patients were enrolled (mean age, 30.3 yrs, range, 23-37 yrs).

Exclusion criteria: smoking, systemic disease, scaling performed or antibiotics taken within 6 months prior to the study, medication potentially affecting the microflora or immune response taken within the previous three months.

In all patients thorough anamnesis and clinical examination were performed. The medical history included periodontal disease in the patient and his closest family, co-existence of systemic diseases, addictions and habits. This examination was the basis for the initial diagnosis and a potential exclusion of patients who did not fulfill the inclusion criteria. 
The study was evaluated and approved by the Ethics Committee of the Medical University of Warsaw and informed consent was obtained from all individuals.

Clinical examination was performed with the use of a constant pressure probe connected to a $\mathrm{PC}$ unit (Florida Probe System, USA) and a standard WHO621 manual probe. The following clinical parameters of periodontal status were measured:

pocket depth (PD) [mm], defined as the distance between the gingival margin and the pocket's bottom, in 6 sites of each tooth; clinical attachment level (CAL) $[\mathrm{mm}]$, defined as the distance between the cementoenamel junction and the pocket's bottom, in 6 sites of each tooth; bleeding index (BI) [\%] according to the simplified scale of Ainamo and Bay (1975), showing the proportion of sites bleeding on probing in all sites measured, in 6 sites of each tooth; plaque index (PI) [\%] according to the simplified scale of O'Leary (1972), showing the relation of surfaces covered with plaque in relation to all surfaces examined, on 4 surfaces of each tooth; ratio of sites with PD exceeding 4mm (\%PD > 4) [\%]. Percentage was calculated by using the Florida Probe system and was included into the analysis.

All parameters except CAL were measured using a CPU-linked probe and verified manually, CAL was measured only with a WHO621 manual probe.

On the day of clinical examinations all patients had dental biofilm composition analysed. Samples of their biofilm were taken with the use of commercially available ready-to-use kits (Perio-Analyse, Institut Clinident, France). Four sites with no suppuration and with the highest PD values in each quadrant were selected and subjected to microbiological analysis. Mean measurements of PD and CAL from all six sites of the teeth examined were further used in statistical calculations and are presented in the results section.

No antiseptic mouthwash was used by the patients for 12 hours before the sampling. Selected teeth were cleaned from supra-gingival plaque and sterile paper points were inserted into the pockets, then they were then left for 30 seconds to collect subgingival plaque. The points with the collected material were placed together in a test-tube and labeled with the code consistent with the code on the patient's card (microbial analysis was performed on the pooled material from each patient - 40 microbiological examinations from 160 samples, 4 from every quadrant of each patient). Each sample prepared in this manner was sent to the manufacturer (Institut Clinident, France).

The quality and quantity of periodontal pathogens was evaluated by means of the real-time PCR technique. In the laboratory, paper points were put in $2 \mathrm{ml}$ sterile tubes and exposed to the factor causing the lysis of bacterial cells. DNA was extracted and purified using specific silica columns by the centrifugation process.
Purified DNA was eluted into $100 \mu$ l of buffer. A PCR mix was prepared for the following bacterial species: Aggregatibacter actinomycetemcomitans, Porphyromonas gingivalis, Tannerella forsythia, Treponema denticola, Prevotella intermedia, Parvimonas micra, Fusobacterium nucleatum, Campylobacter rectus, Eikenella corrodens. The PCR mix included primers/probes, $\mathrm{MgCl}_{2}$ buffer and Taq polymerase. It was distributed to specific tubes for real time PCR. The amount of $2 \mu \mathrm{l}$ of prepared DNA was included in each PCR tube. The reaction was performed on the cycler equipment (Rotor-Gene ${ }^{\circledR} \mathrm{Q}$ thermal cycling system (Qiagen, Germany)). Final quantification was directly visualised and analysed by the cycler's software and compared with the DNA standard and quantification curves. The final quantitative value was calculated for each periodontal bacterium and for the total volume of the sample $(100 \mu \mathrm{l}$, serving as a positive control). The detection rate of bacteria was $10^{2}$.

The study group was divided according to the currently used classification (Armitage, 1999) into two subgroups (gAP and gCP; $n=17$ and $n=23$, respectively), and according to the BGI scale (Offenbacher et al., 2008) also into two subgroups (P2 and P3; $\mathrm{n}=20$ and $n=16$, respectively). Results from both classification systems were analysed independently. Because there were only 4 individuals with diagnosed P1, they were excluded from the BGI scale statistical evaluation.

In each of the four created subgroups minimal, maximal and median values were marked, and using this basis, the normal distribution mean values and standard deviations were also calculated. Due to the fact, that the distribution of values in the study group did not have the features of the Gaussian function, for each division (currently used classification and the BGI scale) the Wilcoxon-Mann-Whitney test was used after the ranking of the studied parameter. The results were considered as statistically significant when in the twotailed test the $\mathrm{p}$ value was lower than 0.05 .

\section{Results}

The results of statistical comparison of the clinical periodontal parameters in the study group divided according to the currently used classification are presented in Table I. In patients with generalized aggressive periodontitis, the mean pocket depth, bleeding index and percentage of pockets deeper than $4 \mathrm{mms}$ were significantly higher than in individuals suffering from chronic periodontitis.

A similar analysis conducted in the group divided according to the BGI scale is presented in Table II. Apart from BI, which by definition has to be higher, other clinical parameters were also significantly higher in the P3 (BGI DL/SB) group. 
Table I

Clinical parameters (mean \pm standard deviation) in the study group divided according to the current classification

\begin{tabular}{|l|c|c|c|c|c|}
\hline & $\mathrm{PD}[\mathrm{mm}]$ & CAL $[\mathrm{mm}]$ & PI [\%] & BI [\%] & $\%$ PD $>4[\%]$ \\
\hline Generalised aggressive periodontitis $(\mathrm{n}=17)$ & $5.38 \pm 1.29$ & $6.73 \pm 1.98$ & $56.4 \pm 25.0$ & $74.1 \pm 16.9$ & $28.6 \pm 20.6$ \\
\hline Generalised chronic periodontitis $(\mathrm{n}=23)$ & $4.09 \pm 1.80$ & $5.28 \pm 2.75$ & $35.0 \pm 30.9$ & $59.7 \pm 30.6$ & $17,8 \pm 19.9$ \\
\hline $\mathrm{p}$ (Wilcoxon-Mann-Whitney) & $\mathrm{p}<0.05$ & $\mathrm{NS}$ & $\mathrm{p}<0.05$ & $\mathrm{NS}$ & $\mathrm{p}<0.05$ \\
\hline
\end{tabular}

Legend: Data is given as means \pm standard deviations. PD - pocket depth; CAL - clinical attachment level; BI - bleeding index; PI - plaque index; NS - not significant.

Table II

Clinical parameters (mean \pm standard deviation) in the study group divided according to the BGI scale

\begin{tabular}{|l|c|c|c|c|c|}
\hline & PD [mm] & CA loss [mm] & BI [\%] & PI [\%] & $\%$ PD $>4[\%]$ \\
\hline P2 $(\mathrm{n}=20)$ & $4.21 \pm 1.32$ & $5.22 \pm 1.82$ & $24.8 \pm 11.7$ & $50.4 \pm 22.0$ & $15.7 \pm 7.5$ \\
\hline P3 (n=16) & $5.51 \pm 1.84$ & $7.15 \pm 2.54$ & $75.5 \pm 16.1$ & $84.9 \pm 15.9$ & $34.1 \pm 27.0$ \\
\hline $\mathrm{p}$ (Wilcoxon-Mann-Whitney) & $\mathrm{p}<0.05$ & $\mathrm{p}<0.05$ & $\mathrm{p}<0.05$ & $\mathrm{p}<0.05$ & $\mathrm{p}<0.05$ \\
\hline
\end{tabular}

Legend: Data is given as means \pm standard deviations; P2 - patients with deep lesions and moderate bleeding (BI between 10 and 50\%); P3 - patients with deep lesions and severe bleeding (BI above 50\%); PD - pocket depth; CAL - clinical attachment level; $\mathrm{BI}$ - bleeding index; PI - plaque index.

Table III

Distribution of patients according to both classifications (currently used and the BGI scale)

\begin{tabular}{|c|c|}
\hline $\begin{array}{c}\text { gAP/P2 } \\
(7 \text { individuals })\end{array}$ & $\begin{array}{c}\text { gAP/P3 } \\
(9 \text { individuals })\end{array}$ \\
\hline $\begin{array}{c}\text { gCP/P2 } \\
(13 \text { individuals })\end{array}$ & $\begin{array}{c}\mathrm{gCP} / \mathrm{P} 3 \\
(7 \text { individuals })\end{array}$ \\
\hline
\end{tabular}

Legend: gAP - generalised aggressive periodontitis; $\mathrm{gCP}$ - generalised chronic periodontitis; P2 - patients with deep lesions (pockets $>4 \mathrm{mms}$ ) and moderate bleeding (BI between 10 and 50\%); P3 - patients with deep lesions (pockets $>4 \mathrm{mms}$ ) and severe bleeding (BI above $50 \%$ ) Note: 1 person with gAP and 3 with gCP were diagnosed with P1 according to BGI scale

Table III shows the number of patients from the study group divided into subgroups according to both evaluated classifications. As the table shows, there was a close to even distribution of individuals among the four possible clinical situations. The only group in which there were more patients than in the others was the group with chronic periodontitis (according to the currently used classification), deep lesions (pockets) and moderate bleeding (according to the BGI scale). However, there was no statistically significant overrepresentation in this subgroup.

The microbial composition of the periodontal pockets in the study group between patients with diagnosis of $\mathrm{AP}$ and CP are presented in Table IV. The Mann-Whitney test revealed the largest difference between those subgroups in the number of C. rectus, and A. actinomycetemcomitans. There were no statistically significant differences in the P. gingivalis, as the mean values would suggest. The reason for this being that the counts of this bacterium were diverse within the study group.
Table $\mathrm{V}$ presents a similar analysis in the study group divided according to the BGI scale. Counts of the C. rectus species were significantly higher in the $\mathrm{P} 3$ group (BGI DL/SB). The differences between other microbiota were not statistically significant.

\section{Discussions}

Periodontal diseases have been repeatedly subjected to different forms of classification. The currently used system, presented at Workshops in 1999 (Armitage, 1999) distinguishes, among others, aggressive and chronic periodontitis. Those two diagnoses underline the variety in dynamics of disease process. According to the American Academy of Periodontology, the characteristic features for aggressive periodontitis are: the loss of clinical attachment at the rate of a minimum of 2 millimeters in 3 months, an excessive inflammatory state when compared with the presence of bacterial biofilm, the lack of systemic diseases and the tendency of hereditary occurrence (Armitage, 1999). The conducted examination confirms these primary features of AP. In patients with a generalized form of the disease there are significantly deeper pockets and a higher bleeding index than in patients with CP. Both parameters describe the intensity of the inflammatory process. A lack of statistical differences in the mean PI (though its value was slightly higher in patients with gAP than with gCP) with coexisting intensified inflammatory reaction is a primary feature of AP. The difference in the percentage of pockets deeper than $4 \mathrm{mms}$ means that in the gAP group intense inflammation is more widely spread. Hypothetically, if patients with localized 
Table IV

Comparison of the number of bacteria in gAP and gCP patients

\begin{tabular}{|l|c|c|c|c|c|}
\hline & \multirow{2}{*}{$\mathrm{gAP}(\mathrm{n}=17)$} & \multirow{2}{*}{$\mathrm{gCP}(\mathrm{n}=23)$} & $\mathrm{p}$ (Wilcoxon-Mann- & \multicolumn{2}{|c|}{ Detectability } \\
\cline { 5 - 6 } & & & Whitney $)$ & $\mathrm{gAP}$ & $\mathrm{gCP}$ \\
\hline A. actinomycetemcomitans & $182.5 \pm 486.5$ & $5.2 \pm 14.5$ & $\mathrm{p}<0.05$ & $8 / 17$ & $4 / 23$ \\
\hline T. forsythia & $1382.4 \pm 1477.0$ & $1267.7 \pm 2298.4$ & $\mathrm{NS}$ & $17 / 17$ & $21 / 23$ \\
\hline C. rectus & $462.9 \pm 365.6$ & $248.3 \pm 318.7$ & $\mathrm{p}<0.05$ & $17 / 17$ & $19 / 23$ \\
\hline T. denticola & $723.2 \pm 959.3$ & $778.7 \pm 1438.6$ & $\mathrm{NS}$ & $15 / 17$ & $21 / 23$ \\
\hline E. corrodens & $312.3 \pm 420.0$ & $231.7 \pm 456.9$ & $\mathrm{NS}$ & $10 / 17$ & $13 / 23$ \\
\hline P. intermedia & $432.2 \pm 1300.1$ & $446.1 \pm 680.9$ & $\mathrm{NS}$ & $9 / 17$ & $17 / 23$ \\
\hline P. micra & $710.4 \pm 1321.9$ & $1861.3 \pm 4595.6$ & $\mathrm{NS}$ & $16 / 17$ & $21 / 23$ \\
\hline P. gingivalis & $1233.1 \pm 2724.6$ & $490.8 \pm 850.9$ & $\mathrm{NS}$ & $11 / 17$ & $15 / 23$ \\
\hline F. nucleatum & $2251.5 \pm 3023.9$ & $1748.5 \pm 2606.4$ & $\mathrm{NS}$ & $17 / 17$ & $23 / 23$ \\
\hline
\end{tabular}

Legend: Data is given as means \pm standard deviations with base value $1=10^{5}$; gAP - patients with generalised aggressive periodontitis; gCP - patients with generalised chronic periodontitis; NS - not significant

Table V

Comparison of the number of bacteria in $\mathrm{P} 2$ and $\mathrm{P} 3$ patients

\begin{tabular}{|l|c|c|c|c|c|}
\hline & \multirow{2}{*}{$\mathrm{P} 2(\mathrm{n}=20)$} & \multirow{2}{*}{$\mathrm{P} 3(\mathrm{n}=16)$} & $\mathrm{p}$ (Wilcoxon-Mann- & \multicolumn{2}{|c|}{ Detectability } \\
\cline { 5 - 6 } & & & Whitney) & $\mathrm{P} 2$ & $\mathrm{P} 3$ \\
\hline A. actinomycetemcomitans & $122.4 \pm 447.4$ & $34.9 \pm 114.6$ & $\mathrm{NS}$ & $6 / 20$ & $4 / 16$ \\
\hline T. forsythia & $1106.5 \pm 1668.4$ & $1748.8 \pm 2463.3$ & $\mathrm{NS}$ & $18 / 20$ & $16 / 16$ \\
\hline C. rectus & $298.0 \pm 357.4$ & $450.4 \pm 350.4$ & $<0.05$ & $18 / 20$ & $16 / 16$ \\
\hline T. denticola & $814.4 \pm 1294.9$ & $796.8 \pm 1331.5$ & $\mathrm{NS}$ & $20 / 20$ & $14 / 16$ \\
\hline E. corrodens & $317.3 \pm 501.5$ & $224.6 \pm 398.8$ & $\mathrm{NS}$ & $11 / 20$ & $9 / 16$ \\
\hline P. intermedia & $150.0 \pm 207.2$ & $877.3 \pm 1442.8$ & $\mathrm{NS}$ & $13 / 20$ & $11 / 16$ \\
\hline P. micra & $678.9 \pm 1373.3$ & $2483.0 \pm 5391.0$ & $\mathrm{NS}$ & $17 / 20$ & $16 / 16$ \\
\hline P. gingivalis & $829.3 \pm 1811.3$ & $940.0 \pm 2243.4$ & $\mathrm{NS}$ & $12 / 20$ & $12 / 16$ \\
\hline F. nucleatum & $1268.6 \pm 1575.2$ & $2908.8 \pm 3831.6$ & $\mathrm{NS}$ & $20 / 20$ & $16 / 16$ \\
\hline
\end{tabular}

Legend: Data is given as means \pm standard deviations with base value $1=10^{5} ; \mathrm{P} 2$ - patients with deep lesions (pockets $>4$ mms) and moderate bleeding (BI between 10 and 50\%); P3 - patients with deep lesions (pockets $>4$ mms) and severe bleeding (BI above 50\%); NS - not significant

forms of both diseases were also included in the study, there could be no difference in the $\% \mathrm{PD}>4$. One must note that the parameters' values in gAP group differ from those observed by Faveri et al. (2008). In Faveri's study mean PD and CAL values from sample sites were higher ( 8.5 and $8.8 \mathrm{mms}$, respectively), the same as percentage of pockets deeper than $4 \mathrm{mms}$ (59.3\%). Plaque index was lower (44.3\%), and bleeding index was higher (71.3\%). The study of Gajardo and coworkers (2005) on a Colombian population reported lower plaque index in gCP group than in our study (48.49\%), but higher bleeding index (54.41\%). PD and CAL parameters were also higher than in our study (7.82 and $7.40 \mathrm{vs}$ 4.09 and 5.28, respectively). There may be at least three explanations for the discrepancy with our results. The geographical factor (East Europe vs South America), higher age of gAP group in our study (30.3 vs 24.1), in comparison with Faveri's study. Moreover, population examined (17 vs 10 in gAP) may be responsible for the bias (Gajardo's numbers of individuals with gAP and gCP were similar to those in present study). The potential role of cultural, ethnic and geographical variables is confirmed by the report of Gjermo et al. (2002), who observed prevalence of severe periodontitis varying from $5.5 \%$ to $50 \%$ in South America only.

The BGI scale is based on the interaction between biofilm and gingival tissues (Offenbacher et al., 2007; 2008). The main weakness of the current and past periodontal disease classifications was that they were grounded on anamnesis and clinical parameters, while it is well known, that the initiation and progress of periodontal tissue destruction have a much deeper basis, with special reference to the imbalance between bacteria and host response, which can be modified by risk factors (Casanova and Abel, 2004). Offenbacher, instead of relying on clinical examination, decided to evaluate 
the microbiological, inflammatory and immunological markers to create biological phenotypes of periodontitis. The next step was to select the clinical parameters that would effectively group patients according to the severity of the disease (Offenbacher et al., 2007). The study confirms that the BGI scale categories (only P2 and P3 studied) seem to be clinically effective, as they create the natural thresholds of disease progression. Selecting patients according to the BGI scale reveals statistically significant differences in all the studied clinical parameters. The difference in $\mathrm{BI}$ is obvious, as it results strictly from the BGI scale categories. Although PD is also associated with the BGI scale, it does not play a role in the differentiation between $\mathrm{P} 2$ and $\mathrm{P} 3$ groups, because in both - by definition - the only condition is that the value of PD in one of the examined sites has to be greater than $4 \mathrm{~mm}$. The PD distinguishes BGI-H and BGI-G from the BGI-DL groups. Therefore, differences in $\mathrm{PD}$ and other clinical parameters between the $\mathrm{P} 2$ and P3 groups support the thesis, that the BGI scale allows to effectively quantify patients according to the severity of the disease. This fact is worth noting due to the simplicity of the scale, which makes it useful for clinicians.

An almost even distribution of individuals in both classifications (as seen in Table III) is the reflection of the fact that they divide patients according to different features. The currently used classification is based on the severity and progress rate of periodontal disease, possibly modified by the general health status, while the BGI scale focuses on the host response to periodontal pathogens. The results of our study suggest that in the course of generalized periodontal inflammation there is no tendency for both classifications to overlap. Whether it is just a unique feature of the studied group or a broader regularity, needs to be further evaluated.

The mean number of A.actinomycetemcomitans in the periodontal pocket was higher in gAP than in $\mathrm{gCP}$, though this observation is not as common as it is supposed to be. While one of the secondary features of aggressive periodontitis is the increased incidence of A.actinomycetemcomitans, reported to reach even 90\% (Armitage, 1999), our research does not fully support this thesis. This pathogen was detected only in 8 out of 17 individuals diagnosed with gAP. Also, there is no conformity in the increased counts of A. actinomycetemcomitans in aggressive periodontitis. While in the present study, regardless of the low detection rate of this species, a statistically significant difference between gAP and gCP groups was found, other reports do not support this finding. Reichert et al. (2009) have evaluated bacterial pathogens in 73 individuals with gAP, 58 patients with gCP and in 69 individuals from their control group. Red complex bacteria and A.actinomycetemcomitans were compared, and no statistical differences were observed. Another study evaluating titers of antibodies in serum also did not reveal any differences between $\mathrm{AP}$ and $\mathrm{CP}$ in A. actinomycetemcomitans and P.gingivalis (Guentsch et al., 2009). Faveri et al. (2008) examined 10 individuals suffering from gAP (Sao Paulo state, Brazil). PCR analysis revealed A. actinomycetemcomitans in 7 of 10 subjects, but $16 \mathrm{~S}$ rRNA cloning did not reveal this pathogen in any of the studied samples. Botero et al. (2007) examined 27 Colombian individuals with gCP and 7 with gAP, and observed higher occurrence of $P$. gingivalis and E.corrodens in subjects with AP. A.actinomycetemcomitans was not detected in any of the patients with diagnosed gAP. It is hypothetically possible that differences in the mentioned reports may result from genetic, ethnic and environmental factors. Thus, microbial features of periodontal diseases (with special attention on aggressive periodontitis) may vary in different geographical regions. Sanz and coworkers (2000) compared 61 adult patients with periodontitis - 30 individuals from Netherlands and 31 from Spain. Microbiological analysis revealed significant differences in the composition of subgingival biofilm. In the Spanish patients there was a high prevalence of $P$. gingivalis and low prevalence of A. actinomycetemcomitans, while the Dutch group was characterized by the high prevalence of A.actinomycetemcomitans and P. micra. The difference in $C$. rectus observed in our patients is similar to that found by Gajardo et al. (2005) in a Chilean study, in which this pathogen was also more frequently observed in AP than in CP (50\% vs. $23.5 \%$, respectively). It was not noted, however, in any of the other mentioned reports (Botero et al., 2007; Faveri et al., 2008; Guentsch et al., 2009; Reichert et al., 2009). Ximenez-Fyvie et al. (2006), while evaluating over 40 bacterial species, didn't observe any differences between gAP and gCP groups, neither in numbers nor in proportions of pathogens. On the other hand, Dogan and coworkers (2003), studying 69 subjects from Turkey, observed higher prevalence of $C$. rectus in gCP than in gAP.

Offenbacher et al. (2007), observed high seropositivity against $C$. rectus in patients from the BGI-G group. This motile Gram-negative rod is able to pass a bloodfaetus barrier and is possibly responsible for premature birth in pregnant women suffering from periodontitis (Madianos et al., 2001). C. rectus belongs to the orange bacterial complex and is connected to gingivitis and the initial stages of periodontitis (Tanner et al., 1998). Based on similarities to another pathogen of the digestive tract, Helicobacter pylori, it has been suggested that C. rectus is able to create ulcerations in the junctional epithelium, leading to epithelial attachment destruction and the initiation of periodontal inflammation (Offenbacher et al., 2007). Moreover, it has been demonstrated that titers of IgG directed against this pathogen were another marker, which distinguished P2 from P3, besides the antibodies against P. gingivalis. Microbiological examination, unlike a measuring of 
antibody titers, revealed that numbers of $P$. gingivalis in $\mathrm{P} 2$ and $\mathrm{P} 3$ groups were significantly higher than in the BGI-G group, but the differences between the P2 and P3 groups were not observed (Offenbacher et al., 2007). Our present study did not confirm those differences and the mean values of this pathogen in both subgroups were similar. It could be caused by the initial patient selection criteria, as only generalized periodontitis was examined. In the present study, the BGI scale reveals differences only in the $C$. rectus species, while the currently used classification also shows it in A. actinomycetemcomitans. This may be considered as a weakness of the BGI scale. On the other hand, in the present study both classifications seem to be independent and almost equally divide patients. gAP does not have a tendency to be overrepresented in the $\mathrm{P} 2$ or $\mathrm{P} 3$ groups, as individuals with gAP diagnosis are equally distributed in both. That - together with a low detection rate of A. actinomycetemcomitans in study groups - may be the reason for differences in the pathogen counts between the P2 and $\mathrm{P} 3$ groups. Low detection rates regarded also other species - E. corrodens and P. intermedia, and none of the two studied classifications made their numbers statistically differ between the subgroups of the patients. In the next study Offenbacher et al. (2008) observed an increased incidence of the orange complex bacteria, which correlated with an increased severity of the inflammatory process. Our study confirmed those results. In patients from the P3 group the total orange complex bacteria counts were almost threefold higher than in the P2 group (data not shown in tables; $6.7 \times 10^{8}$ vs. $2.3 \times 10^{8}$ respectively, differences statistically significant).

Sampling in the present study was limited to 4 sites from 4 selected teeth in each patient, while in the literature there are reports where each tooth (Torrungruang et al., 2009), or 4 sites around each tooth (Socransky et al., 1998) were sampled. There is the necessity to confirm the results obtained in our study with more thorough bacterial examination.

Concluding: the currently used classification system for periodontal diseases based on the rate of attachment loss is difficult to be applied in the daily office, also microbiological tests are not always unambiguous. Therefore the BGI scale based on PD and BI has every chance to become an effective tool in the diagnostics of periodontal diseases.

\section{Literature}

Ainamo J. and I. Bay. 1975. Problems and proposals for recording gingivitis and plaque. Int. Dent. .J 25: 229-235.

Armitage G.C. 1999. Development of a classification system for periodontal diseases and conditions. Ann. Periodontol. 4: 1-6.

Botero J.E., A. Contreras, G. Lafaurie, A. Jaramillo, M. Betancourt and R.M. Arce. 2007. Occurrence of periodontopathic and superinfecting bacteria in chronic and aggressive periodontitis subjects in a Colombian population. J. Periodontol. 78: 696-704.

Casanova J.L. and L. Abel. 2004. The human model: a genetic dissection of immunity to infection in natural conditions. Nat. Rev. Immunol. 4: 55-66.

Dogan B., J. Antinheimo, D. Cetiner, A. Bodur, G. Emingil, E. Buduneli, C. Uygur, E. Firatli, L. Lakio and S. Asikainen. 2003. Subgingival microflora in Turkish patients with periodontitis. J. Periodontol. 74: 803-814.

Faveri M., M.P. Mayer, M. Feres, L.C. de Figueiredo, F.E. Dewhirst and B.J. Paster. 2008. Microbiological diversity of generalized aggressive periodontitis by $16 \mathrm{~S}$ rRNA clonal analysis. Oral. Microbiol. Immunol. 23: 112-118.

Gajardo M., N. Silva, L. Gomez, R. Leon, B. Parra, A. Contreras and J. Gamonal. 2005. Prevalence of periodontopathic bacteria in aggressive periodontitis patients in a Chilean population. J. Periodontol. 76: 289-294.

Gjermo P., C.K. Rösing, C. Susin and R. Oppermann. 2002. Periodontal diseases in Central and South America. Periodontol. 2000. 29: 70-78.

Guentsch A., M. Puklo, P.M. Preshaw, E. Glockmann, W. Pfister, J. Potempa and S. Eick S. 2009. Neutrophils in chronic and aggressive periodontitis in interaction with Porphyromonas gingivalis and Aggregatibacter actinomycetemcomitans. J. Periodont. Res. 44: 368-377.

Heller D., C.M. Silva-Boghossian, R.M. Do Souto and A.P. Colombo. 2012. Subgingival microbial profiles of generalized aggressive and chronic periodontal diseases. Arch. Oral. Biol. 57: 973-980. Madianos P.N., S. Lieff, A.P. Murtha, K.A. Boggess, R.L.Jr. Auten, J.D. Beck and S. Offenbacher. 2001. Maternal periodontitis and prematurity. Part II: maternal infection and fetal exposure. Ann. Periodontol. 6: 175-182.

O'Leary T.J., R.B. Drake and J.E. Naylor. 1972. The plaque control record. J. Periodontol. 43: 38.

Offenbacher S., S.P. Barros, R.E. Singer, K. Moss, R.C. Williams and J.D. Beck. 2007. Periodontal disease at the biofilm-gingival interface. J. Periodontol. 78: 1911-1925.

Offenbacher S., S.P. Barros and J.D. Beck. 2008. Rethinking periodontal inflammation. J. Periodontol. 79 (suppl. 1): 1577-1584.

Reichert S., H.K.G. Machulla, J. Klapproth, U. Zimmermann, Y. Reichert, C. Glaser, H.G. Schaller and S. Schulz. 2009. Interleukin-2 - 330 and 166 gene polymorphisms in relation to aggressive or chronic periodontitis and the presence of periodontopathogenic bacteria. J. Periodont. Res. 44: 628-635.

Rylev M. and M. Kilian. 2008. Prevalence and distribution of principal periodontal pathogens worldwide. J. Clin. Periodontol. 35 (Suppl. 8): 346-361.

Sanz M., A.J. van Winkelhoff, D. Herrera, N. Dellemijn-Kippuw, R. Simon and E. Winkel. 2000. Differences in the composition of the subgingival microbiota of two periodontitis populations of different geographical origin. A comparison between Spain and The Netherlands. Eur. J. Oral. Sci. 108: 383-392.

Socransky S.S., A.D. Haffajee, M.A. Cugini, C. Smith and R.L.Jr. Kent.1998. Microbial complexes in subgingival plaque. J. Clin Periodontol. 25: 134-144.

Tanner A., M.F. Maiden, P.J. Macuch, L.L. Murray and R.L. Jr. Kent. 1998. Microbiota of health, gingivitis and initial periodontitis. J. Clin. Periodontol. 25: 85-98.

Torrungruang K., P. Bandhaya, K. Likittanasombat and C. Grittayaphong. 2009. Relationship between the presence of certain bacterial pathogens and periodontal status of urban Thai adults. J. Periodontol. 80: 122-129.

Ximenez-Fyvie L.A., A. Almaguer-Flores, V. Jacobo-Soto, M. LaraCordoba, J.Y. Moreno-Borjas and E. Alcantara-Maruri. 2006. Subgingival microbiota of periodontally untreated Mexican subjects with generalized aggressive periodontitis. J. Clin. Periodontol. 33: 869-877. 
